\title{
Lesión intracraneal transorbitaria por florete. Caso clínico
}

\author{
A. Calvo-Rubal***; F. Martínez**** y A. Tarigo* \\ *Servicio de Neurocirugía del Hospital Maciel. Ministerio de Salud Pública. ** Servicio de Neurocirugía. Banco de Seguros del Estado. \\ ***Departamento de Anatomía. Facultad de Medicina. Montevideo. Uruguay.
}

\section{Resumen}

Introducción. Las heridas penetrantes intracraneales por arma blanca son poco frecuentes en países occidentales y su diagnóstico y tratamiento pueden presentar dificultades que las hacen diferentes al resto de los traumatismos de cráneo.

Caso clínico. Se presenta el caso de un profesor de esgrima que practicando sin la debida protección, recibió una lesión por florete, transorbitaria derecha con penetración intracraneana. La misma le produjo un hematoma intraparenquimatoso temporoparietal derecho, con efecto de masa. Dicha lesión se hizo evidente en la evolución, ya que de inicio, el paciente no presentó ningún síntoma oftalmológico o neurológico. El hematoma se evacuó sin incidentes con buena evolución clínica del paciente. No hubo lesiones en el globo ocular.

Discusión y conclusiones. La lesión de estructuras intracraneales por objetos penetrantes transorbitarios es poco frecuente. Su diagnóstico exige un alto índice de sospecha, teniendo en cuenta el objeto agresor, mas allá del estado clínico inicial del paciente.

PALABRAS CLAVE: Traumatismo craneal penetrante. Traumatismo orbitario. Hematoma intracerebral traumático.

Cranial wounds of the skull by fencing - foil

\section{Summary}

Penetrating stab cranial wounds of the skull by fencing - foil are rare in western countries.

Case report. This 46 year old man suffered a penetrating stab wound of the skull throughout the right orbital region. As a consecuence he developed on intracranial hematoma requiring surgical evacuation.

Discussion and conclusions. Damage of intracranial cuntents due to transorbital penetrating objects other than missiles is a rare event.

Recibido:19-08-05. Aceptado: 20-11-05
KEY WORDS: Head injury. Penetrating skull injury. Stab wounds. Orbital trauma. Intracrial hematoma.

\section{Introducción}

Las heridas penetrantes intracraneales por arma blanca o cuerpos extraños diferentes a los proyectiles de arma de fuego son muy poco frecuentes ${ }^{4,12,19,26}$.

La entrada del agente lesivo puede ser a través de la calota o de la base del cráneo en cualquiera de sus secto$\mathrm{res}^{4,13}$. Dada la constitución anatómica y las relaciones de la base del cráneo, la penetración se puede hacer también a través de la órbita, la boca, las fosas nasales o partes blandas de la cara ${ }^{9,18-20,25,27,28,30}$.

Frecuentemente, la lesión intracraneal no es diagnosticada de inicio, sobre todo cuando el paciente no tiene síntomas neurológicos. Pero teniendo en cuenta el tipo de agresión con un objeto capaz de atravesar el cráneo, se debe tener un alto índice de sospecha para diagnosticarlas y evitar secuelas diferidas potencialmente graves o mortales ${ }^{9,10,25}$.

A continuación reportamos un caso de lesión intra-craneal con entrada en órbita, causada por un florete durante la práctica de esgrima.

\section{Caso clínico}

Paciente varón de 46 años, zurdo, instructor de esgrima que el 23/7/04, mientras daba clases a un adolescente, sin la debida protección de la máscara reglamentaria, recibe una herida con florete "mudo" (solo con la protección de un pequeño botón metálico en la punta) en la región orbitaria derecha. Es visto por médico de emergencia móvil quien encuentra un paciente con examen neurológico normal, sin herida penetrante en globo ocular y sin alteraciones de la oculomotricidad o la agudeza visual, por lo que indica tratamiento sintomático.

En las primeras 24 horas de evolución, se añadieron cefaleas, vómitos, intenso dolor en órbita derecha y exoftalmos. Posteriormente presenta disminución del estado de conciencia, ingresando en un centro hospitalario donde se procede a su sedación e intubación, realizándose 


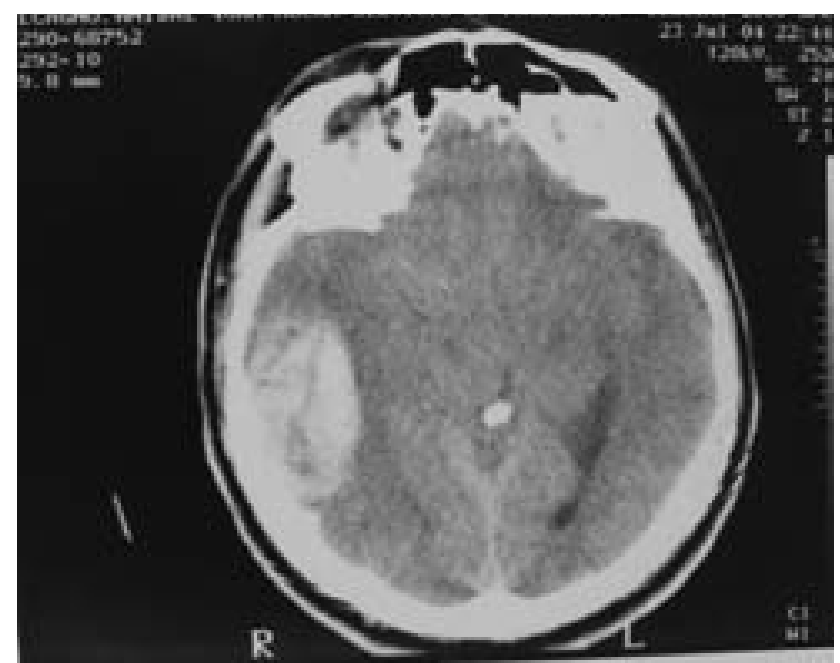

Figura 1. TC de cráneo que muestra el hematoma intraparenquimatoso temporoparietal derecho. Se ve un ligero desplazamiento de la calcificación de la glándula pineal hacia la izquierda.

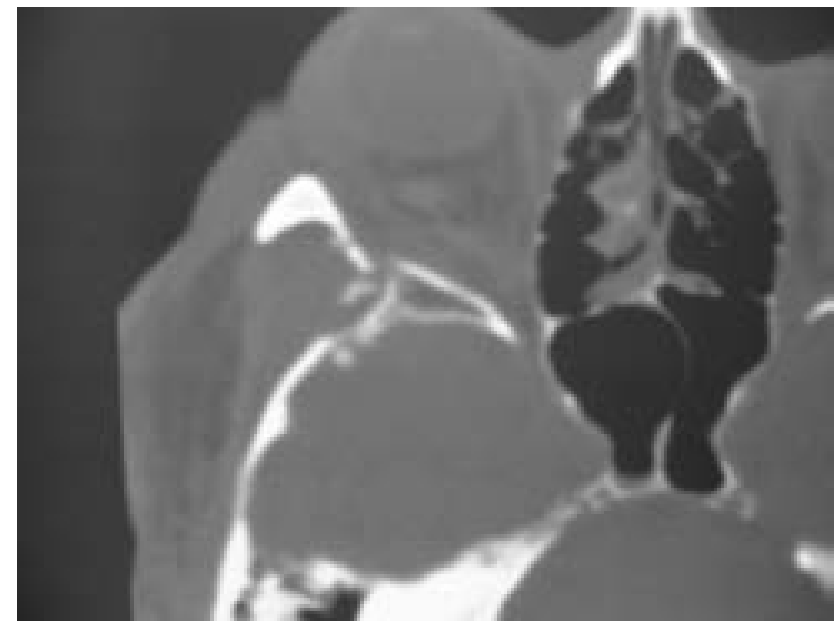

Figura 2. TC de cráneo, ventana ósea. Se evidencia la fractura en la pared externa de la órbita y del sector anterior de la fosa temporal, que marcan el trayecto seguido por el florete.

una tomografía craneana (TC).

La misma (figura 1) mostró un hematoma lobar temporoparietal derecho, de franco predominio en el primer lóbulo, con lámina subdural laminar asociado. La lesión ejercía efecto de masa, con desplazamiento de la línea media a la izquierda. El examen clínico mostraba indemnidad de globo ocular .

En la ventana ósea se constató que la hoja del florete, tras lesionar el sector posterior de la pared externa de la órbita, pasó a través de la fosa temporal y ala mayor del esfenoides, penetrando luego en la fosa craneana media (Figura 2). En la figura 3 se muestra en una pieza anatómica, el trayecto seguido por el arma lesiva.
2006; 17: 550-554

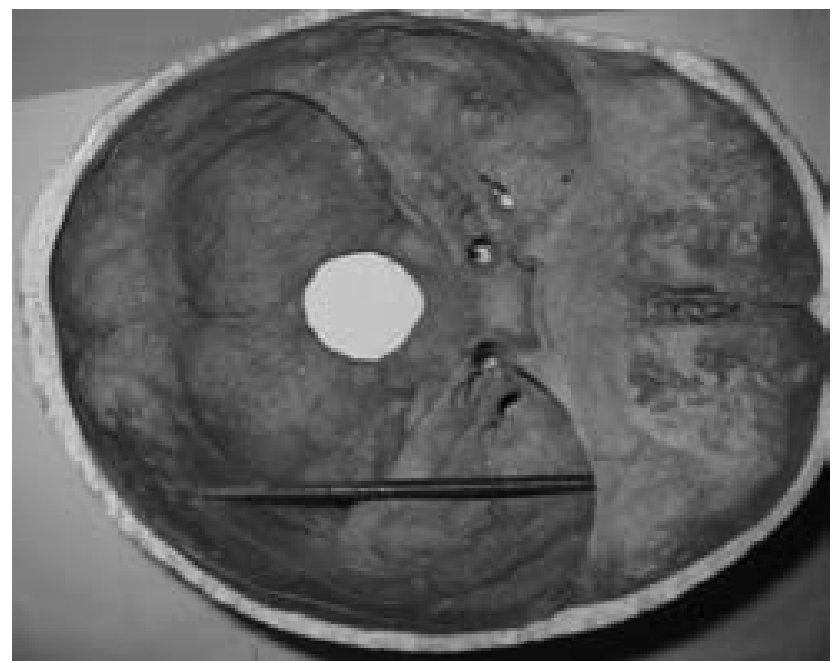

Figura 3. Pieza anatómica que muestra el trayecto seguido por el florete en su penetración intracraneana.

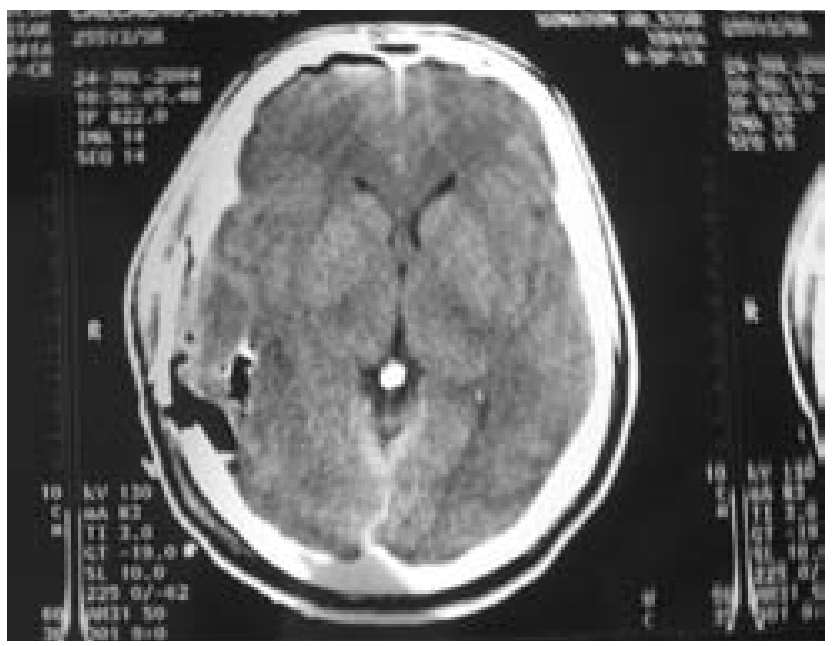

Figura 4. TC de cráneo postoperatoria. Se ve la correcta evacuación del hematoma y la realineación de la calcificación de la glándula pineal.

El paciente se intervino de urgencia en la madrugada del 24/7/04, mediante colgajo de Walker derecho. Al levantar el colgajo óseo, la duramadre estaba tensa y azulada; se abrió la misma aspirándose el hematoma subdural agudo. Posteriormente, se hizo una incisión cortical en la segunda circunvolución temporal (T2), para evacuar el hematoma intraparenquimatoso. No se identificó lesión de grandes vasos; se colocó un hemoclip en una arteriola en el sector posterior de la diéresis. Se cerró, dejando un catéter para medir la presión intracraneana (PIC).

La TC de control postoperatoria (Figura 4) mostró buena evacuación del hematoma, con discreto "swelling" del hemisferio, pero con PIC dentro de rangos normales.

En la evolución clínica, presentó un despertar excitado, por lo que se postergó su extubación por 48 horas. La 
misma se pudo realizar luego sin incidentes.

En control previo al alta, estaba con una puntuación de 15 en la escala de coma de Glasgow (GCS) y sin elementos focales. En ojo derecho, se evidenciaba una visión normal con una discreta hemorragia subconjuntival.

En el último control en policlínica (2/9/04) estaba asintomático y reintegrado a sus tareas habituales.

\section{Discusión}

Las heridas penetrantes intracraneales no producidas por armas de fuego, son muy poco frecuentes. El objeto lesivo, punto de entrada al cráneo, el tipo y motivo de la lesión son variables ${ }^{12,27}$. La causa de la lesión puede responder a accidentes, agresiones, intentos de suicidio o ejecuciones. Los agentes lesivos descritos van desde machetes o cuchillos hasta lápices, palillos de comida china, clavos, arpones, agujas, lapiceras, trozos de madera o de plás$\operatorname{tico}^{5,6,9,12,13,17,19,20,22-24,26,27,30,31}$.

El área de ingreso al cráneo también es variable y se describen varios sitios probables, como la calota en cualquiera de sus sectores, base del cráneo, la órbita, cavidad bucal, fosas nasales o partes blandas de la cara $4,6,18,27,30$.

El ingreso de un objeto punzante por la órbita asociado a lesión intracraneal es poco frecuente y dentro de este tipo de lesiones, se debe mencionar por su importancia histórica la de Enrique II de Francia (1529-1559). Éste recibió, durante un torneo de pista, una lesión de lanza por parte de Montgomery, capitán de su guardia. Montgomery atravesó el yelmo de oro de Enrique II, produciéndole una grave lesión temporal izquierda con entrada en órbita, que pocos días después le produciría la muerte. Más recientemente, en las finales de florete de las olimpíadas de Estocolmo hubo una lesión similar con muerte de uno de los competidores, lo que llevó a cambiar las reglas de protección.

En Uruguay, la extensión de los duelos llevó a formular una ley que los reglamentó en agosto de 1920 y que fue anulada en $1992^{8}$.

Los duelos se realizaban con pistola de "avant-carga" o con sable. Para ello había que tener un entrenamiento en la escuela de armas. De esta forma, en determinados estratos sociales, particularmente políticos, militares, periodistas o profesionales y a pesar de los prejuicios religiosos, se difundió el arte de la esgrima que luego declinó ${ }^{3,8}$. Actualmente su práctica continúa llevándose a cabo, con la protección adecuada, como la careta de fibra y entramado metálico, el peto de "keblar" y el florete eléctrico ${ }^{14}$.

En cuanto al tipo de lesión, se pueden reconocer dos grupos: penetrantes o perforantes. Estos últimos son mucho menos frecuentes, pero más graves, ya que implican frecuentemente lesiones bihemisféricas y transventriculares. A su vez, al atravesar varios compartimentos del cráneo, tienen más probabilidad de producir lesiones vasculares ${ }^{1,12}$.
Ahora, refiriéndose específicamente a las lesiones intracraneales con entrada orbitaria, cuando el objeto punzante atraviesa la órbita, lo más frecuente es que penetre en el cráneo a través del techo de esta última por dos razones: 1) el techo orbitario es delgado y, por lo tanto, poco resistente y 2) el individuo, para evitar la lesión, de forma refleja extiende la cabeza en un esfuerzo por alejarla del arma lesiva. De esta forma, le expone a la misma el techo de la órbita. Por la disposición de las estructuras cerebrales en relación con la base del cráneo, la región cerebral mas lesionada con este punto de entrada es el lóbulo frontal ${ }^{5,19}$. De forma mucho menos frecuente, el objeto podrá penetrar a través de la hendidura esfenoidal, el conducto óptico o la pared externa de la órbita ${ }^{19,21}$, lo que ocurrió en el caso que presentamos.

Las estructuras lesionadas en estas vías de entrada también son diferentes: seno cavernoso y eventualmente tronco encefálico o cerebelo (entrada en la hendidura esfenoidal), nervio óptico, si se compromete el canal óptico, y fosa o lóbulo temporal si la lesión se hace a través de la pared externa de la órbita (Figuras 3 a, b y c) 4,18,19,21,24.

Dado que los objetos lesivos pueden ser de diversa naturaleza (metal, madera o plástico) y muchas veces el sitio de entrada es puntiforme, se debe tener un alto índice de sospecha frente a estas lesiones para descubrir el daño intracraneal $^{21,29}$. En nuestro caso, el paciente fue visto por un médico de urgencia, que halló un paciente asintomático y sin lesión ocular evidente; pero a las horas comenzó con síntomas de hipertensión endocraneal que llevaron a su estudio con una TC de cráneo, que evidenció la lesión cerebral.

Si la penetración intracraneal pasa desapercibida inicialmente y no ha producido lesiones de entidad, puede manifestarse de forma tardía bajo la forma de abscesos cerebrales, seudoaneurismas, infartos por espasmo o trombosis, fístulas arteriovenosas o carótido-cavernosas ${ }^{15,24,28}$. Los procesos infecciosos se ven de forma mas frecuente en lesiones periorbitarias y por objetos de madera con restos retenidos ${ }^{5,22,28}$. Pueden presentarse en corto plazo luego del trauma o varios años después, por lo que algunos autores recomiendan la exploración y remoción de restos del objeto, aún en ausencia de síntomas ${ }^{22}$. Los restos de madera pueden ser muy difíciles de identificar mediante estudios de imagen, particularmente la radiografía simple o la TC, ya que la madera es radiolúcida ${ }^{19}$. Si hay un alto índice de sospecha, frente a lesiones con objetos de madera, debería solicitarse una RM, ya que aumenta el rendimiento diagnóstico ${ }^{19}$. Solomon y col $^{29}$ recomiendan solicitar la TC de cráneo con cortes coronales si se sospecha firmemente la penetración intracraneal, a fin de detectar las fracturas del techo orbitario.

Es discutible el uso rutinario de angiografía de los 4 vasos de cuello en este tipo de lesiones ${ }^{16}$. Algunos autores plantean hacerla, por el alto número de lesiones vasculares asociadas a lesiones penetrantes: hasta 35\% según Kieck y 
$\operatorname{col}^{15}$. Se recomendaría hacer la angiografía en toda lesión penetrante que tenga un trayecto en relación con un vaso arterial importante y de preferencia, en la primer semana ${ }^{15}$. Los tipos de lesiones vasculares descritas son seudoaneurismas, secciones totales o parciales, fístula carótido-cavernosa o arteriovenosas durales, trombosis o vasoespasmo grave por hemorragia meníngea ${ }^{7,15}$. En nuestro caso no se solicitó arteriografía, ya que no se evidenció ninguna lesión vascular en el trayecto del florete en la cirugía.

\section{Bibliografía}

1. Arabi, B.: Management of traumatic aneurysms caused by high-velocity missile head wounds. Neurosurg Clin NA 1995; 6: 775-797

2. Arunkumar, R.J., Selvapandian, S., Rajshekhar, V.: Penetrating intracranial wooden object: case report and review of CT morphology, complications, and management. Surg Neurol 1999; 51: 617-620.

3. Baldick, R.: The duel history. New York; Barnes \& Noble, 1996.

4. Bauer, M., Patzelt, D.: Intracranial stab injuries: case report and case study. Forensic Sci Int 2002; 129: 122-127 (Abstract).

5. Bert, F., Ouahes, O., Lambert-Zechowsky, N.: Brain abscess due to Bacillus macerans following penetrating periorbital injury. J Clin Microbiol 1995; 33: 1950-1953.

6. Bhatjiwale, M.G., Goel, A., Muzumdar, D.P.: Transnasal intracranial entry of a flying wire fragment. Br J Neurosurg 2001; 15: 256-258.

7. Bullock, R., van Dellen, J.R.: Acute carotid-cavernous fistula with retained knife blade after transorbital stab wound. Surg Neurol 1985; 24: 555-558

8. Coral Luzzi, P.: Código de honor con las leyes relativas al duelo. Montevideo, 1950.

9. Davis, G.A., Holmes, A.D., Klug, G.L.: Delayed presentation of transorbital intracranial pen. J Clin Neurosci 2000; 7 : 545-548.

10. Dinakaran, S., Noble, P.J.: Silent orbitocranial penetration by a pencil. J Accid Emerg Med 1998; 15: 274-275.

11. De Souza Reilly, J.J.: Caras y Caretas. Bs As, 1936.

12. Fernández-Melo, R., Morán, A.F., López-Flores, G., Bouza-Molinas M., García-Maeso, I., Benavides-Barbosas, J.: Trauma craneoencefálico perforante por arpón. Presentación de un caso y revisión de la literatura. Neurocirugía 2002; 13:397-400.

13 Gluncic, I., Roje, Z., Tudor, M., Gluncic, V.: Unusual stab wound of the temporal region. Croat Med J 2001; 42 :579-582.

14. Guerreño, F.: Esgrima. Método de enseñanza de florete, espada y sable. Talleres gráficos STETIP. Buenos Aires, 1972.

15. Kieck, C.F., De Villiers, J.C.: Vascular lesions due to transcranial stab wounds. J Neurosurg 1984; 60: 42-46.

16. Kitakami, A., Kirikae, M., Kuroda, K., Ogawa, A.: Transorbital-transpetrosal penetrating cerebellar injury: case report. Neurol Med Chir (Tokyo) 1999; 39: 150-152 (Abstract).
2006; 17: 550-554

17. Lee, J.S., Lee, J.E., Oum, B.S., Cha, S.H.: Orbitocranial injury caused by wood. Korean J Ophtalmol 1999; 13: 128-132.

18. Lunetta, P., Ohberg, A., Sajantila, A.: Suicide by intracerebellar ballpoint pen. Am J Forensic Med Pathol 2002; 23: 334-337 (Abstract).

19 Matsumoto, S., Hasuo, K., Mizushima, A., Mihara, F., Fukui, M., Shirouzu, T., Masuda, K.: Intracranial penetrating injuries via the optic canal. Am J Neuroradiol 1998; 19: 1163-1165.

20. Matsuyama, T., Okuchi, K., Nogami, K., Hata, M., Murao, Y.: Transorbital penetrating injury by a chopstick: case report. Neurol Med Chir (Tokyo) 2001; 41: 345-348 (Abstract).

21. Mono, J., Hollenberg, R.D., Harvey, J.T.: Occult intracranial transorbital penetrating injuries Ann Emerg Med 1986; 15: 589-591.

22. Nishio, Y., Hayashi, N., Hamada, H., Hirashima, I., Endo, S.: A case of delayed brain abscess due to a retained intracranial wooden foreign body: a case report and review of the last 20 years. Acta Neurochir (Wien) 2004; 146: 847-850.

23. Rahman, N.U., Jamjoom, A., Jamjoom, Z.A., Abu el-Asrar, A.: Orbito-cranial injury caused by penetrating metallic foreign bodies: report of two cases. Int Ophthalmol 1997; 21: 13-17.

24. Rezai, A.R., Lee, M., Kite, C., Smyth, D., Jafar, J.J.: Traumatic posterior cerebral artery aneurysm secondary to an intracranial nail: case report. Surg Neurol 1994; 42: 312-315.

25. Rompen, J.C., Meek, M.F., van Andel, M.V.: A cause celebre: the so-called "ballpoint murder". J Forensic Sci. 2000; 45: 1144-1147 (Abstract).

26. Salar, G., Costella, G.B., Mottaran, R., Mattana, M., Gazzola, L., Munari, M.: Multiple cranicerebral injuries from penetrating nails. Case illustration. J Neusosurg 2004; 100: 963.

27. Sandoval, H., Lobato, R.D., Domínguez, J., Ortega, J.M., Sarabia, R.: Trauma craneoencefálico penetrante por objeto de madera. Neurocirugía 1992; 3: 158-160.

28. Shih, T.Y., Kuo, Y.L.: Development of intracranial complications following transoral stab wounds in children. Report of two cases. Pediatr. Neurosurg. 2002; 37: 35-37 (Abstract).

29. Solomon, K.D., Pearson, P.A., Tetz, M.R., Baker, R.S.: Cranial injury from unsuspected penetrating orbital trauma: a review of five cases. J. Trauma. 1993; 34: 285-289.

30. Takanashi, Y., Shinonaga, M., Manaka, H.: Penetrating brain injury with nasal entry by a plastic stick. Case report. J. Neurosurg. Sci. 2002; 46: 25-27.

31. Tancioni, F., Gaetani, P., Pugliese, R., Rodríguez y Baena, R.: Intracranial nail. A case report. J. Neurosurg. Sci. 1994; 38: 239-243.

Calvo-Rubal, A.; Martínez, F.; Tarigo, A.: Lesión intracraneal transorbitaria por florete. Caso clínico. Neurocirugía 2006; 17: 550-554.

Correspondencia postal: F. Martínez Benia. Missisipi 1536 Block D. Apto 501 entre Rivera y Pilcomayo. Malvin. Montevideo. Uruguay. 


\section{Comentario al trabajo Lesión intracraneal transorbitaria por florete. Caso clínico de A. Calvo-Rubal y cols}

En el presente trabajo los autores ilustran un caso que resalta uno de los problemas más importantes de los traumatismos craneales perforantes: la alta probabilidad de lesiones intracraneales en presencia de lesiones superficiales mínimas o no evidenciables en la exploración hace preciso en la evaluación de estos pacientes complementar la exploración clínica con estudios radiológicos. Estas lesiones pueden manifestarse de forma diferida a pesar de una ausencia inicial de síntomas ${ }^{1}$, como queda claramente ilustrado en este caso. Otro problema es la posibilidad de lesiones vasculares intracraneales. En este caso, la exploración intraoperatoria hizo descartar a los autores la sospecha de estas lesiones al hallarse el trayecto de la herida separado de estructuras vasculares importantes. No existe evidencia que permita decidir en qué pacientes se debe complementar el estudio con una arteriografía cerebral aunque recomendaciones (con grado de "opción") indican la conveniencia de realizarla en pacientes en los que la trayectoria de la herida pasa. a través o cerca de la cisura de Silvio, cerca de la carótida supraclinoidea o cerca del seno cavernoso u otros senos venosos. También se recomienda en el caso de desarrollo de hematoma diferido o HSA no explicada ${ }^{2}$. Un ejemplo de esta última posibilidad fue presentado en el IX congreso de la SENEC (Alén, J.F., Lagares, A., C'ampollo, J., Miranda, P., Ramos, A., Rivas, J.J.: Tratamiento de aneurismas cerebrales mediante "stents" recubiertos. IX Congreso de la SENEC, Santander):

Un varón de 21 años sufrió una agresión con un destornillador en la órbita izquierda, presentando amaurosis por ese ojo, exoftalmos y oftalmoplejia. Se realizó una TAC craneal diagnosticando HSA de predominio supraselar y en el inicio de la cisterna silviana izquierda (Figura 1). La arteriografía del día de ingreso mostró una pequeña lesión en cara anterior de carótida en su segmento suprapetroso sugerente de pseudoaneurisma traumático (Figura 2). Se repitió la arteriografía 3 días después donde se demostró el aumento de tamaño de dicho pseudoaneurisma y el desarrollo de fístula directa al seno cavernoso. La lesión fue tratada mediante la colocación de un "stent" recubierto a dicho nivel comprobándose la ausencia de relleno en la angiografía tras el procedimiento, Tres meses después el paciente continuaba con paresia del III par, había recuperado el déficit del VI y veía luces y bultos a 2 metros con el ojo afecto. En la arteriografía de control, la carótida se encontraba permeable, sin relleno del pseudoaneurisma ni de la fístula.

En el caso comentado en el presente artículo los autores decidieron no completar el estudio con una arteriografía. Tal vez se podría haber indicado dicha prueba basándonos en la proximidad a los vasos silvianos y el desarrollo del hematoma, pero con las recomendaciones anteriores cualquiera de las dos actitudes podría ser válida en este paciente.

\section{Bibliografía}

1. Gudeman, S.K., Young, H.F., Miller, JD., Ward, J.D., Becker, D.P.: Indications for operative treatment and operative technique on closed head injury. En Becker, D.P, Gudeman, S.K (ed). Textbook of head injury. Philadelphia; Saunders, 1989: pp 138-181.

2. Neuroimaging in the management of penetrating brain injury. J Trauma 2001; 51(suppl): 7-11.

\section{A. Pérez Núñez J.F. Alén \\ Madrid}

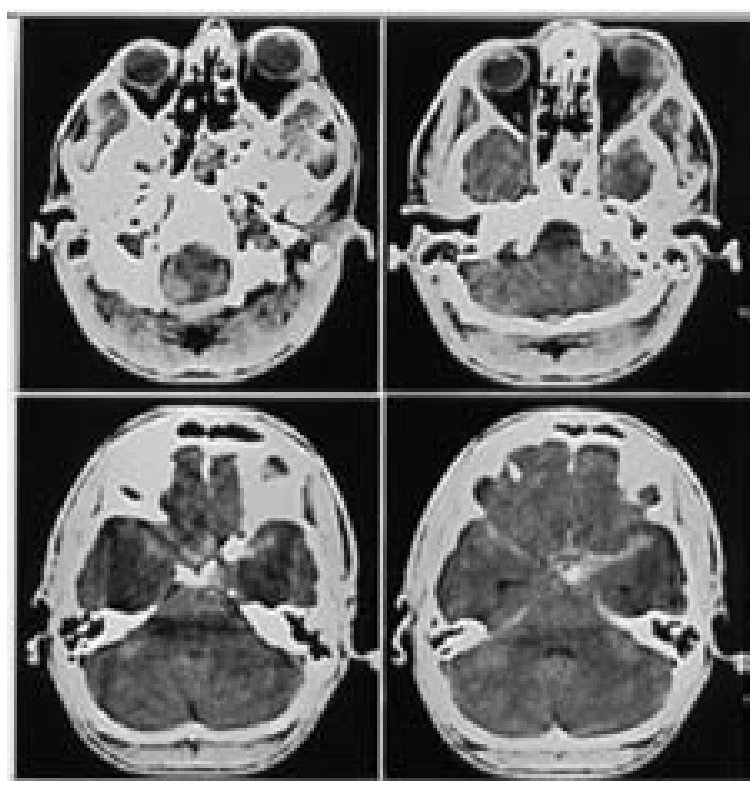

Figura 1. TC craneal que muestra la presencia de hemorragia subaracnoidea en las cisternas de la base craneal y en el inicio de la cisterna silviana izquierda.

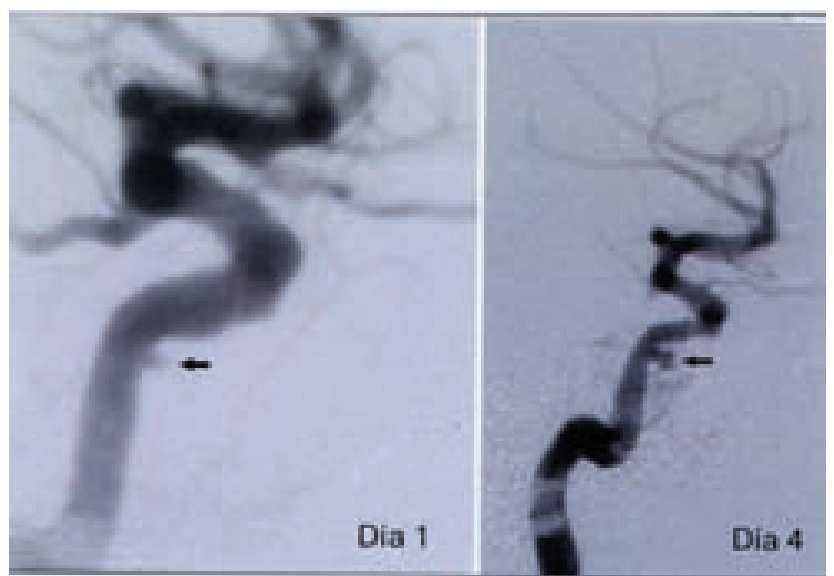

Figura 2. Arteriografia donde se observa la presencia de una pequeña lesión pseudoaneurismática carotídea el día del ingreso (izquierda) y su posterior crecimiento en el estudio realizado tres días más tarde (derecha). 